\title{
Matrix Presentation of Uncertainties Propagation in the Realization of ITS-90 Temperature Scale using Standard Platinum Resistance Thermometers
}

\author{
Rudolf Palenčár ${ }^{1}$, Stanislav Ďuriš ${ }^{1}$, Jakub Palenčár ${ }^{1}$, Martin Halaj ${ }^{1}$, Lubomír Šooš², \\ ${ }^{1}$ Institute of Automation, Measurement and Applied Informatics, Faculty of Mechanical Engineering, Slovak University of \\ Technology, Nám. slobody 17, 81231 Bratislava, Slovak Republic, rudolf.palencar@stuba.sk \\ ${ }^{2}$ Institute of Manufacturing Systems, Environmental Technology and Quality Management, Faculty of Mechanical \\ Engineering, Slovak University of Technology, Nám. slobody 17, 81231 Bratislava, Slovak Republic
}

\begin{abstract}
The paper presents a matrix approach to the propagation of uncertainties in the realization of the ITS-90 using Standard Platinum Resistance Thermometers (SPRT) calibrated at Defining Fixed Points (DFPs). The procedure allows correlations to be included between SPRT resistances measured during the calibration at the DFPs (i.e., the realization of the ITS-90) and the resistances measured during the subsequent use of the SPRT to measure temperature $T_{90}$. The example also shows the possible contribution of these correlations to the overall temperature uncertainty measured by a calibrated SPRT.
\end{abstract}

Keywords: Uncertainty, correlation, standard platinum resistance thermometer (SPRT), ITS-90, matrix algebra.

\section{INTRODUCTION}

Evaluation of the propagation of uncertainties in the realization of the 1990 temperature scale (ITS-90) [22] using the Standard Platinum Resistance Thermometer (SPRT) calibrated at Defining Fixed Points (DFP) is a problem that can be solved in various ways. Literature [1] gives an overview of the different approaches. BIPM document [2] is based on orthogonal interpolation functions. This approach is mentioned in [3], [4], [5], [6]. Other approaches are based on GUM [7], [8]. Part of the approaches (see also [2], [9]) presents the uncertainty propagation for SPRT with summation formulas [10], [11], [12], [13], [14], [15], [16], [17]. In accordance with GUM Supplement 2 [8], we present a matrix algebra approach. The authors have already presented a partial matrix approach in [18], [19], but only for estimating the parameters of the deviation equation. The GUM algebraic approach was used to determine uncertainties [7]. In accordance with the GUM Supplement 2 [8], the literature [9] introduces propagation of temperature scale uncertainties in a matrix form. The matrix approach becomes more elegant than the algebraic approach. However, in [9] correlations between resistances in DFPs are not considered. The resistances in DFPs alone can be correlated due to the same conditions in the laboratory both in the realization of the DFP and in the temperature measurement, as shown in [12]. Using data obtained from the Slovak Institute of Metrology
(SMU), literature [12] gives an example of the determination of correlation coefficients and thus covariance between individual DFPs in the SPRT calibration. As analyzed in [20], consideration of covariance can lead to a reduction in the uncertainty of the temperature scale realization in the calibration laboratory. This is important for the realization of the temperature scale at the highest (primary) level directly in the calibration laboratory. The actual procedure for evaluating uncertainties, based directly on the GUM [7], considering correlations between resistances in DFPs using summation relationships, is elaborated in [10], [11], [12], [13]. Those documents show the possible meaning of including covariance in the calculations. A matrix approach to consider correlations between resistances in DFPs was also presented at the $\mathrm{XX}^{\text {th }}$ IMEKO World Congress [21].

This paper describes the procedure for determining uncertainties considering correlations between input quantities in matrix form. It follows the procedure outlined in [9] and extends it to the possibility of including covariations between DFPs in determining the temperature uncertainty measured by a calibrated SPRT in DFP. The procedure is based directly on the GUM [7] and the GUM Supplement 2 [8]. The uncertainties of SPRT resistances $R\left(\mathrm{DFP}_{i}\right)$ in the calibration and SPRT resistances $R_{\mathrm{t}}$ in use, as well as covariances between them, are assumed. The uncertainties due to different types of non-uniqueness are not covered here. These issues are addressed in [2], [16]. 


\section{MODEL OF SPRT CALIBRATION}

The inverse reference equation is applied for the calculation of temperatures $T$ when measured by SPRT according to ITS90 [22]

$$
T=\left.W_{\mathrm{r}}^{-1}\right|_{W_{\mathrm{r}}}
$$

Function $W_{\mathrm{r}}$, considered in the paper, is defined as

$$
W_{\mathrm{r}}=W-\sum_{i=1}^{N} a_{i} f_{i}(W)
$$

where

$$
W=\frac{R}{R_{\mathrm{TPW}}}
$$

and $R$ is the SPRT resistance at temperature $T ; R_{\text {TPW }}$ is SPRT resistance in TPW; $f_{i}(W)$ are functions given for individual sub-ranges of temperatures in [20], $a_{i}$ are coefficients of respective deviation equations, obtained by SPRT calibration.

When calibrating SPRTs, the laboratory determines resistance values in DFP, their uncertainties and covariances between them.

Vector $\boldsymbol{R}_{\text {cal }}$ contains resistance values measured in DFP

$$
\boldsymbol{R}_{\mathrm{cal}}=\left(R_{\mathrm{DFP} 1}, R_{\mathrm{DFP} 2}, \cdots, R_{\mathrm{DFPN}}, R_{\mathrm{TPW} 1}, R_{\mathrm{TPW} 2}, \cdots, R_{\mathrm{TPW} N}\right)^{\mathrm{T}}
$$

and the respective covariance matrix is

$$
\boldsymbol{U}\left(\boldsymbol{R}_{\text {cal }}\right)=\left(\begin{array}{cccc}
u^{2}\left(R_{\mathrm{DFP} 1}\right) & u\left(R_{\mathrm{DFP} 1}, R_{\mathrm{DFP} 2}\right) & \cdots & u\left(R_{\mathrm{DFP} 1}, R_{\mathrm{TPW} N}\right) \\
u\left(R_{\mathrm{DFP} 2}, R_{\mathrm{DFP} 1}\right) & u^{2}\left(R_{\mathrm{DFP1} 1}\right) & \cdots & u\left(R_{\mathrm{DFP} 2}, R_{\mathrm{TPW} N}\right) \\
\vdots & \vdots & \ddots & \vdots \\
u\left(R_{\mathrm{TPW} N}, R_{\mathrm{DFP} 1}\right) & u\left(R_{\mathrm{TPW} N}, R_{\mathrm{DFP} 2}\right) & \cdots & u^{2}\left(R_{\mathrm{TPW} N}\right)
\end{array}\right)
$$

where $R_{\mathrm{DFP} i}$ is the SPRT resistance at the temperature of $\mathrm{DFP}_{i}$; $R_{\mathrm{TPW} i}$ is the SPRT resistance at the temperature of TPW measured after the measurement of resistance in $\mathrm{DFP}_{i}$; $u\left(R_{\mathrm{DFP} i}\right)$ is the standard uncertainty of the resistance at the temperature of $\mathrm{DFP}_{i} ; u\left(R_{\mathrm{DFP} i}, R_{\mathrm{DFP} j}\right)$ is the covariance between SPRT resistances in $\mathrm{DFP}_{i}$ and $\mathrm{DFP}_{j}$.

Matrix notation can be used to calculate the coefficients of the deviation equation. If the relation (2) is applied for $N$ fixed points, it will be

$$
\left(\begin{array}{c}
W_{\mathrm{r}, \mathrm{DFP} 1}-W_{\mathrm{DFP} 1} \\
\vdots \\
W_{\mathrm{r}, \mathrm{DFPN}}-W_{\mathrm{DFPN}}
\end{array}\right)=\left(\begin{array}{ccc}
f_{1}\left(W_{\mathrm{DFP} 1}\right) & \ldots & f_{N}\left(W_{\mathrm{DFP} 1}\right) \\
\vdots & \ddots & \vdots \\
f_{N}\left(W_{\mathrm{DFPN}}\right) & \ldots & f_{N}\left(W_{\mathrm{DFPN}}\right)
\end{array}\right)\left(\begin{array}{c}
a_{1} \\
\vdots \\
a_{N}
\end{array}\right)
$$

where $W_{\mathrm{DFP} i}$ for $I=1,2, \ldots, N$ are relative resistances in respective $\mathrm{DFP}_{i}$; $W_{\mathrm{r}, \mathrm{DFP} i}$ are given by ITS-90 [13]. Values of $W_{\mathrm{DFP} i}$ are determined using equation (3) for $R=R_{\mathrm{DFP} i}$ and $R_{\mathrm{TPW}}=R_{\mathrm{TPW} i}$.

If we denote $W_{\mathrm{r}, \mathrm{DFP} i}-W_{\mathrm{DFP} i}=\Delta W_{\mathrm{DFP} i}$, equation (6) can be written as

$$
\Delta \boldsymbol{W}_{\mathrm{DFP}}=\boldsymbol{M}_{\mathrm{DFP}} \boldsymbol{a}
$$

where $\Delta \boldsymbol{W}_{\mathrm{DFP}}$ is the vector of dimension $N ; \boldsymbol{M}_{\mathrm{DFP}}$ is the matrix of dimension $N \times N$ and $\boldsymbol{a}$ is the vector of coefficients of the deviation equation of dimension $N$.

As $\boldsymbol{M}_{\mathrm{DFP}}{ }^{-1}$ exists, coefficients of the deviation equation will be

$$
\boldsymbol{a}=\boldsymbol{M}_{\mathrm{DFP}}^{-1} \Delta \boldsymbol{W}_{\mathrm{DFP}}
$$

\section{UNCERTAINTY PROPAGATION IN USING A CALIBRATED SPRT TO MEASURE TEMPERATURE $T_{90}$}

\subsection{Uncertainty of the temperature $T$}

The temperature uncertainty calculated according to (1) is given by

$$
u(T)=\left.\frac{\partial T}{\partial W_{\mathrm{r}}(T)}\right|_{W_{\mathrm{r}}} u\left(W_{\mathrm{r}}\right) .
$$

\subsection{Uncertainty of reference resistance ratio at the temperature $T$}

In order to determine the uncertainty $u\left(W_{\mathrm{r}}\right)$, model (2) will be written in a matrix form according to [7]

$$
W_{\mathrm{r}}-W+\boldsymbol{a}^{\mathrm{T}} \boldsymbol{f}(W)=0 .
$$

According to [8, paragraph 6.3.1] represents a multivariate function, which specifies the relationship of a set of output quantities $\boldsymbol{Y}=\left(Y_{1}, Y_{2}, \cdots, Y_{m}\right)^{\mathrm{T}}$ and input quantities $\boldsymbol{X}=$ $\left(X_{1}, X_{2}, \cdots, X_{n}\right)^{\mathrm{T}}$. Generally, such a function is written as

$$
\boldsymbol{h}(\boldsymbol{X}, \boldsymbol{Y})=\mathbf{0}, \quad \boldsymbol{h}=\left(h_{1}, h_{2}, \cdots, h_{m}\right)^{\mathrm{T}}
$$

Given an estimate $\boldsymbol{x}$ of $\boldsymbol{X}$, an estimate $\boldsymbol{y}$ of $\boldsymbol{Y}$ is given by the solution of the system of equation [8]

$$
h(x, y)=0
$$

According to [8], equation (11) for the covariance matrix $\boldsymbol{U}(\boldsymbol{y})$ of vector $\boldsymbol{y}$ holds

$$
\boldsymbol{U}(\boldsymbol{y})=\boldsymbol{C} \boldsymbol{U}_{\boldsymbol{x}} \boldsymbol{C}^{\mathrm{T}}
$$

where according to [8, chapter 6] (assuming existence of $\boldsymbol{C}_{y}^{-\mathbf{1}}$ )

$$
C=-C_{y}^{-1} C_{x}
$$

and $\boldsymbol{C}_{y}$ is the sensitivity matrix of dimension $m \times m$ containing the partial derivatives $\partial h_{l} / \partial Y_{j}, l=1, \cdots m, j=$ $1, \cdots, m$ and $\boldsymbol{C}_{\boldsymbol{x}}$ is the sensitivity matrix of dimension $m \times N$ containing the partial derivatives $\partial h_{l} / \partial X_{i}, l=1, \cdots m, i=$ $1, \cdots, N$, all derivatives being evaluated at $\boldsymbol{X}=\boldsymbol{x}$ and $\boldsymbol{Y}=\boldsymbol{y}$.

Considering that in equation (14) there is one output variable $\boldsymbol{Y}=W_{\mathrm{r}}$ and the vector of input quantities is $\boldsymbol{X}=$ $\left(W, a_{1}, \cdots, a_{N}\right)^{\mathrm{T}}$, it will be (if we denote the estimate and also the quantity $W_{\mathrm{r}}$ as $W_{\mathrm{r}}$, as well as estimates and also quantities as $a_{i}$ )

where

$$
u^{2}\left(W_{\mathrm{r}}\right)=\boldsymbol{c}^{\mathrm{T}} \boldsymbol{U}_{W, \boldsymbol{a}} \boldsymbol{c},
$$

$$
\boldsymbol{c}^{\mathrm{T}}=-\boldsymbol{C}_{y}^{-1} \boldsymbol{C}_{x}
$$


and

$$
\boldsymbol{C}_{y}=1
$$

$$
\boldsymbol{C}_{x}=\left(-1+\sum_{i=1}^{N} a_{i} \frac{\partial f_{i}(W)}{\partial W}, f_{1}(W), f_{2}(W), \cdots, f_{N}(W)\right)
$$

then

$$
\boldsymbol{c}^{\top}=\left(1-\sum_{i=1}^{N} a_{i} \frac{\partial f_{i}(W)}{\partial W},-f_{1}(W),-f_{2}(W), \cdots,-f_{N}(W)\right)
$$

Further, because $W_{\mathrm{r}} \approx W$, the derivative $\frac{\partial W_{\mathrm{r}}}{\partial W} \approx 1$ and the expression $1-\sum_{i=1}^{N} a_{i} \frac{\partial f_{i}(W)}{\partial W} \approx 1$. Specific shapes of the sensitivity coefficient matrices $\boldsymbol{c}^{\mathrm{T}}$ for the individual ITS-90 sub-ranges are given in [8].

3.3. The covariance matrix $\boldsymbol{U}_{W, \boldsymbol{a}}$ of the relative resistance vector and the coefficients of the SPRT deviation equation

Determination of the covariance matrix $\boldsymbol{U}_{W, \boldsymbol{a}}$ in equation (15) is again based on implicate model (11), where

$$
\boldsymbol{Y}=\left(W, a_{1}, \cdots, a_{N}\right)^{\mathrm{T}}=\left(W, \boldsymbol{a}^{\mathrm{T}}\right)^{\mathrm{T}}
$$

and

$$
\begin{gathered}
\boldsymbol{X}=\left(R, R_{\mathrm{TPW}}, W_{\mathrm{DFP} 1}, W_{\mathrm{DFP} 2}, \cdots, W_{\mathrm{DFPN}}\right)^{\mathrm{T}} \\
=\left(\boldsymbol{R}_{\text {meas }}^{\mathrm{T}}, \boldsymbol{W}_{\mathrm{DFP}}^{\mathrm{T}}\right)^{\mathrm{T}} .
\end{gathered}
$$

Taking equations (3), (6), and (7) into consideration, the evaluation model gets the form

$$
\left(\begin{array}{c}
W-\frac{R}{R_{\mathrm{TPW}}} \\
\boldsymbol{\Delta} \boldsymbol{W}_{\mathrm{DFP}}
\end{array}\right)+\left(\begin{array}{c}
\mathbf{0}_{1 \times N} \\
\boldsymbol{M}_{\mathrm{DFP}, N \times N},
\end{array}\right) \boldsymbol{a}_{N \times 1}=\mathbf{0}_{(1+N) \times 1}
$$

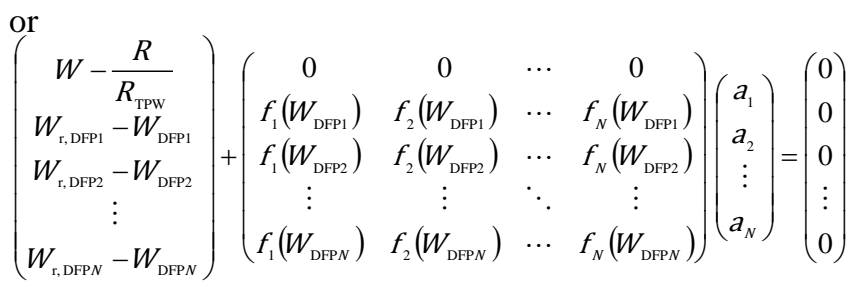

Covariance matrix $\boldsymbol{U}_{W, \boldsymbol{a}}$ according to equation (13) will be

$$
\boldsymbol{U}_{W, \boldsymbol{a}}=\boldsymbol{C} \boldsymbol{U}_{\boldsymbol{R}_{\mathrm{meas}}, \boldsymbol{W}_{\mathrm{DFP}}} \boldsymbol{C}^{\mathrm{T}}
$$

The matrix $\boldsymbol{C}$ is given by (14). Sensitivity coefficient matrices for model (21) will be in the form (see also [9], equations (12) and (13))

$$
C_{x}=\left(\begin{array}{cccccc}
-\frac{1}{R_{\mathrm{TPW}}} & \frac{R}{R_{\mathrm{TPW}}^{2}} & 0 & 0 & \cdots & 0 \\
0 & 0 & -1+\sum_{i=1}^{N} a_{i} \frac{\partial f_{i}}{\partial W_{\mathrm{DPP} 1}} & 0 & \cdots & 0 \\
0 & 0 & 0 & -1+\sum_{i=1}^{N} a_{i} \frac{\partial f_{i}}{\partial W_{\mathrm{DFP} 2}} & \cdots & 0 \\
\vdots & \vdots & \vdots & \vdots & \ddots & \vdots \\
0 & 0 & 0 & 0 & \cdots & -1+\sum_{i=1}^{N} a_{i} \frac{\partial f_{i}}{\partial W_{\mathrm{DPPN}}}
\end{array}\right)
$$

and

$$
C_{y}=\left(\begin{array}{ccccc}
0 & 0 & 0 & \cdots & 0 \\
0 & f_{1}\left(W_{\mathrm{DFP}}\right) & f_{2}\left(W_{\mathrm{DFP1}}\right) & \cdots & f_{N}\left(W_{\mathrm{DFP1}}\right) \\
0 & f_{1}\left(W_{\mathrm{DPP} 2}\right) & f_{2}\left(W_{\mathrm{DFP} 2}\right) & \cdots & f_{N}\left(W_{\mathrm{DFP} 2}\right) \\
\vdots & \vdots & \vdots & \ddots & \vdots \\
0 & f_{1}\left(W_{\mathrm{DFPN}}\right) & f_{2}\left(W_{\mathrm{DPPN}}\right) & \cdots & f_{N}\left(W_{\mathrm{DFPN}}\right)
\end{array}\right)
$$

The specific shapes of the sensitivity coefficient matrices $\boldsymbol{C}_{\boldsymbol{y}}$ and $\boldsymbol{C}_{\boldsymbol{x}}$ for the individual sub-ranges of ITS-90 are presented in [9].

3.4. Covariance matrix $\boldsymbol{U}_{\boldsymbol{R}_{\text {meas }}, \boldsymbol{W}_{D F P}}$ of the resistances vector when measuring the temperature and the relative resistances in DFP when calibrating SPRT

In order to determine the covariance matrix $\boldsymbol{U}_{\boldsymbol{R}_{\text {meas }}, \boldsymbol{W}_{\mathrm{DFP}}}$ in equation (22), we start again from the implicit model in the form (11), where the vector of output variables is

$$
\begin{aligned}
& \boldsymbol{Y}=\left(R_{\text {out }}, R_{\mathrm{TPW} \text { out }}, W_{\mathrm{DFP} 1}, W_{\mathrm{DFP} 2}, \cdots, W_{\mathrm{DFPN}}\right)^{\top}= \\
& \left(\boldsymbol{R}_{\text {meas out }}^{\top}, \boldsymbol{W}_{\mathrm{DFP}}^{\top}\right)^{\top}
\end{aligned}
$$

and vector of input quantities is

$$
\begin{aligned}
& \boldsymbol{X}=\left(R_{\mathrm{in}}, R_{\mathrm{TPW} \text { in }}, R_{\mathrm{DFP} 1}, \cdots, R_{\mathrm{DFPN}}, R_{\mathrm{TPW} 1}, \cdots, R_{\mathrm{TPWN}}\right)^{\top}= \\
& \left(\boldsymbol{R}_{\text {meas in }}^{\top}, \boldsymbol{R}_{\text {cal }}^{\top}\right)^{\top},
\end{aligned}
$$

where $\boldsymbol{R}_{\text {meas in }}^{\mathrm{T}}=\left(R_{\mathrm{in}}, R_{\mathrm{TPW} \text { in }}\right) \quad$ are resistances when measuring temperature and $\boldsymbol{R}_{\mathrm{cal}}^{\mathrm{T}}=\left(R_{\mathrm{DFP} 1}\right.$, $\left.\cdots, R_{\mathrm{DFPN}}, R_{\mathrm{TPW} 1}, \cdots, R_{\mathrm{TPWN}}\right)$ are resistances obtained during calibration.

The introduction of resistances $R_{\mathrm{in}}, R_{\mathrm{TPW}}$ in and $R_{\text {out }}, R_{\mathrm{TPW} \text { out }}$ allows considering resistances $R, R_{\mathrm{TPW}}$ as input and output quantities and thus introduces the possibility of considering covariances between resistors $R, R_{\mathrm{TPW}}$ when measuring temperature and resistances in DFP during calibration (e.g., when using resistance in TPW obtained during calibration, as shown in Part 4).

The model of evaluation will be in the form of equations (3)

$$
\left(\begin{array}{c}
R_{\text {out }}-R_{\text {in }} \\
R_{\text {TPW out }}-R_{\text {TPW in }} \\
W_{\text {DFP1 }}-\frac{R_{\mathrm{DFP1}}}{R_{\mathrm{TPW} 1}} \\
W_{\mathrm{DFP2} 2}-\frac{R_{\mathrm{DFP} 2}}{R_{\mathrm{TPW} 2}} \\
\vdots \\
W_{\mathrm{DFPN} N}-\frac{R_{\mathrm{DFPN}}}{R_{\mathrm{TPWN}}}
\end{array}\right)=\left(\begin{array}{c}
0 \\
0 \\
0 \\
0 \\
\vdots \\
0
\end{array}\right)
$$


Covariance matrix $\boldsymbol{U}_{\boldsymbol{R}_{\text {meas }}, \boldsymbol{W}_{\text {DFP }}}$ according to the equation (13) will be

$$
\boldsymbol{U}_{\boldsymbol{R}_{\text {meas }}, \boldsymbol{W}_{\mathrm{DFP}}}=\boldsymbol{C} \boldsymbol{U}_{\boldsymbol{R}_{\text {meas in }}, \boldsymbol{R}_{\mathrm{cal}}} \boldsymbol{C}^{\mathrm{T}}
$$

Matrix $\boldsymbol{C}$ is defined by equation (14). Sensitivity coefficient matrix $\boldsymbol{C}_{y}$ of the $(2+N) \times(2+N)$ type for model (25) has the shape

$$
C_{y}=I
$$

where $\boldsymbol{I}$ is unit matrix.

Sensitivity coefficient matrices $\boldsymbol{C}_{x}$ of the $(2+N) \times(2+$ $2 N$ ) type for model (25) have the shape

$$
C_{x}=-\left(\begin{array}{cccccccc}
1 & 0 & 0 & \cdots & 0 & 0 & \cdots & 0 \\
0 & 1 & 0 & \cdots & 0 & 0 & \cdots & 0 \\
0 & 0 & \frac{1}{R_{\mathrm{TPW} 1}} & \cdots & 0 & -\frac{R_{\mathrm{DFP} 1}}{R_{\mathrm{TPW} 1}^{2}} & \cdots & 0 \\
\vdots & \vdots & \vdots & \ddots & \cdots & \cdots & \ddots & \cdots \\
0 & 0 & 0 & \cdots & \frac{1}{R_{\mathrm{TPW} N}} & 0 & \cdots & -\frac{R_{\mathrm{DFP} N}}{R_{\mathrm{TPW} \mathrm{N}}^{2}}
\end{array}\right)
$$

Matrix $C$

$$
\boldsymbol{C}=\left(\begin{array}{cccccccc}
1 & 0 & 0 & \cdots & 0 & 0 & \cdots & 0 \\
0 & 1 & 0 & \cdots & 0 & 0 & \cdots & 0 \\
0 & 0 & \frac{1}{R_{\mathrm{TPW} 1}} & \cdots & 0 & -\frac{R_{\mathrm{DFP} 1}}{R_{\mathrm{TPW} 1}^{2}} & \cdots & 0 \\
\vdots & \vdots & \vdots & \ddots & \cdots & \cdots & \ddots & \cdots \\
0 & 0 & 0 & \cdots & \frac{1}{R_{\mathrm{TPW} N}} & 0 & \cdots & -\frac{R_{\mathrm{DFP} N}}{R_{\mathrm{TPW} N}^{2}}
\end{array}\right)
$$

Let us also denote $\boldsymbol{U}_{\boldsymbol{R}}=\boldsymbol{U}_{\boldsymbol{R}_{\text {meas in }}, \boldsymbol{R}_{\text {cal }}}$, then

$\boldsymbol{U}_{R}=\left(\begin{array}{ccccc}u^{2}(R) & u\left(R, R_{\mathrm{TPW}}\right) & u\left(R, R_{\mathrm{DFP} 1}\right) & \cdots & u\left(R, R_{\mathrm{TPW} N}\right) \\ u\left(R_{\mathrm{TPW}}, R\right) & u^{2}\left(R_{\mathrm{TPW}}\right) & u\left(R_{\mathrm{TPW}}, R_{\mathrm{DFP}}\right) & \cdots & u\left(R_{\mathrm{TPW}}, R_{\mathrm{TPW} N}\right) \\ u\left(R_{\mathrm{DFP} 1}, R\right) & u\left(R_{\mathrm{DFP1}}, R_{\mathrm{TPW}}\right) & u^{2}\left(R_{\mathrm{DFP1}}\right) & \cdots & u\left(R_{\mathrm{DFP1}}, R_{\mathrm{TPW} N}\right) \\ \vdots & \vdots & \vdots & \ddots & \vdots \\ u\left(R_{\mathrm{TPW} N}, R\right) & u\left(R_{\mathrm{TPW} N}, R_{\mathrm{TPW}}\right) & u\left(R_{\mathrm{TPW} N}, R_{\mathrm{DFP} 1}\right) & \cdots & u^{2}\left(R_{\mathrm{TPW} N}\right)\end{array}\right)$

If we denote

$$
\boldsymbol{U}_{\boldsymbol{R}_{\text {meas }}}=\left(\begin{array}{cc}
u^{2}(R) & u\left(R, R_{\mathrm{TPW}}\right) \\
u\left(R, R_{\mathrm{TPW}}\right) & u^{2}\left(R_{\mathrm{TPW}}\right)
\end{array}\right),
$$

$\boldsymbol{U}\left(\boldsymbol{R}_{\text {meas in }}, \boldsymbol{R}_{\mathrm{cal}}\right)=\left(\begin{array}{cccc}u\left(R, R_{\mathrm{DFP} 1}\right) & u\left(R, R_{\mathrm{DFP2} 2}\right) & \cdots & u\left(R, R_{\mathrm{TPW} N}\right) \\ u\left(R_{\mathrm{TPW}}, R_{\mathrm{DFP} 1}\right) & u\left(R_{\mathrm{TPW}}, R_{\mathrm{DPP} 2}\right) & \cdots & u\left(R_{\mathrm{TPW}}, R_{\mathrm{TPW} N}\right)\end{array}\right)$,

Then

$$
\boldsymbol{U}_{\boldsymbol{R}}=\left(\begin{array}{cc}
\boldsymbol{U}_{\boldsymbol{R}_{\text {mes }}} & \boldsymbol{U}\left(\boldsymbol{R}_{\text {meas }}, \boldsymbol{R}_{\text {cal }}\right) \\
\boldsymbol{U}\left(\boldsymbol{R}_{\text {meas }}, \boldsymbol{R}_{\text {cal }}\right)^{\mathrm{T}} & \boldsymbol{U}_{\boldsymbol{R}_{\text {cal }}}
\end{array}\right)
$$

Here, the covariance matrix $\boldsymbol{U}_{\boldsymbol{R}_{\text {meas in }}}$ of the $2 \times 2$ type contains the uncertainties of SPRT resistance measurements at $T$ and at TPW and covariances between them. The covariance matrix $\boldsymbol{U}\left(\boldsymbol{R}_{\text {cal }}\right)$ of the $2 N \times 2 N$ type contains the uncertainties of SPRT resistances measurement in DFP and the covariances between them during the calibration of SPRT, relationship (5). The $2 \times 2 N$ covariance matrix $\boldsymbol{U}\left(\boldsymbol{R}_{\text {meas in, }} \boldsymbol{R}_{\text {cal }}\right)$ contains the covariances between SPRT resistances measurement when measuring temperature $T$ and during SPRT calibration.

The covariance matrix $\boldsymbol{U}_{\boldsymbol{R}}$ of the type $(2+2 N) \times(2+2 N)$, i.e. uncertainties of SPRT resistances in DFP during calibration of SPRT and SPRT resistances when measuring temperature $T$, is determined by calibrating SPRT based on uncertainties and covariance budget and analysis of conditions when measuring temperature by calibrated SPRT (see also [2] and [15], [18]).

For some considerations, it is also preferable to define a random vector correlation matrix. The correlation matrix $\boldsymbol{R}_{\boldsymbol{X}}$ of the $m$ dimensional vector $\boldsymbol{X}$ is of the $\mathrm{m} \times \mathrm{m}$ type. Diagonal elements have magnitude one while non-diagonal elements represent correlation coefficients

$$
r\left(X_{i}, X_{j}\right)=\frac{u\left(X_{i}, X_{j}\right)}{u\left(X_{i}\right) u\left(X_{j}\right)}
$$

It also applies

$$
\boldsymbol{U}_{\boldsymbol{X}}=\boldsymbol{P}_{\boldsymbol{X}} \boldsymbol{R}_{\boldsymbol{X}} \boldsymbol{P}_{\boldsymbol{X}} \text { and } \boldsymbol{R}_{\boldsymbol{X}}=\boldsymbol{P}_{\boldsymbol{X}}^{-1} \boldsymbol{U}_{\boldsymbol{X}} \boldsymbol{P}_{\boldsymbol{X}}^{-1}
$$

where $\boldsymbol{P}_{\boldsymbol{X}}$ is a diagonal matrix of dimension $m \times m$ with diagonal elements $u\left(x_{1}\right), u\left(x_{2}\right), \cdots, u\left(x_{m}\right)$. addressed in [2], [16].

\section{COVARIANCE MATRIX OF RESISTANCES WHEN MEASURING TEMPERATURE AND DURING CALIBRATION}

As shown in [18], measurements of resistances in DFP during calibration can be correlated. Assuming that the output signal is measured using a resistance bridge, the components entering the uncertainty calculation are caused by the following effects:

- chemical impurities of the substance in the DFP,

- hydrostatic-head effect (corresponding to the location of SPRT sensing element in DFP)

- self heating effect (error) of the SPRT,

- immersion effect (error) of the SPRT,

- effect of gas pressure in the (metal) DFPs,

- choice of fixed point value from plateau,

- isotopic variations (for TPW only),

- residual gas pressure in the TPW cell,

- changes of resistances of standard resistor caused by changing of its temperature that is measured,

- non-linearity of the resistance bridge,

- uncertainty of calibration of resistance standard. 
There may be a situation where common influences occur when measuring resistance in temperature measurement and calibration in a calibration laboratory; then also resistance $R$ can be correlated with resistances $R_{\mathrm{DFP} i}$ and $R_{\mathrm{TPW}}$.

\subsection{Temperature measurement in a calibration laboratory}

When measuring the temperature by calibrated SPRT in a calibration laboratory, this is typically the case when one SPRT resistance in the TPW is used or one TPW cell is used to measure the SPRT resistance in the TPW during both calibration and measurement. The case of one resistance in TPW is the case of calibration in sub-ranges up to $0^{\circ} \mathrm{C}$. For temperatures above $0{ }^{\circ} \mathrm{C}$, resistance in TPW is measured after the measurement of the SPRT resistance in each $\mathrm{DFP}_{i}$. Then, for the calculation of the resistance in TPW, we take the arithmetic mean of the resistances in $\mathrm{TPW}_{i}$ and the uncertainty of the TPW and the covariances between the resistance in the TPW and the individual resistances in $\mathrm{DFP}_{i}$ as their arithmetic mean.

\section{a) All resistances are non-correlated and one TPW is used} for calibration

First, let us consider the case that all measurements and DFPs and other impacts on SPRT are non-correlated, the laboratory uses independent DFPs, independent resistive bridges are used to measure all resistances (both realizations and measurements as well as other influences), i.e. all covariations are zero.

Correlation matrix of resistances of input quantities $\boldsymbol{R}_{\boldsymbol{R}}$ of the $(2+N) \times(2+N)$ type is

$$
\boldsymbol{R}_{\boldsymbol{R}}=\boldsymbol{I}_{(2+N) \times(2+N)}
$$

b) Resistances in calibration are correlated and one TPW is used for calibration

Correlation matrix of resistances of input quantities $\boldsymbol{R}_{\boldsymbol{R}}$ of the $(2+N) \times(2+N)$ type is

$$
\boldsymbol{R}_{R}=\left(\begin{array}{lllll}
1 & r\left(R, R_{\mathrm{TPW}}\right) & r\left(R, R_{\mathrm{TPW} 1}\right) & \cdots & r\left(R, R_{\mathrm{TPW} N}\right) \\
r\left(R_{\mathrm{TPW}}, R\right) & 1 & r\left(R_{\mathrm{TPW}}, R_{\mathrm{DFP} 1}\right) & \cdots & r\left(R_{\mathrm{TPW}}, R_{\mathrm{DFP} N}\right) \\
r\left(R_{\mathrm{DFP} 1}, R\right) & r\left(R_{\mathrm{DFP} 1}, R_{\mathrm{TPW}}\right) & 1 & \cdots & r\left(R_{\mathrm{DFP} 1}, R_{\mathrm{DFP} N}\right) \\
\vdots & \vdots & \ddots & \ddots & \vdots \\
r\left(R_{\mathrm{DFP}}, R\right) & r\left(R_{\mathrm{DFP} N}, R_{\mathrm{TPW}}\right) & r\left(R_{\mathrm{DFP} N}, R_{\mathrm{DFP} 1}\right) & \cdots & 1
\end{array}\right)
$$

4.2 Temperature measurement outside the calibration laboratory

Measurement outside the calibration laboratory means here that the client uses their own TPW and their own resistance bridge. So, the covariances between $R_{\mathrm{TPW}}$ out and resistances in DFPs will be zero. If the client uses the SPRT resistance in TPW, it will be evaluated as in the calibration laboratory except for the resistance measurements at the measured temperature. a) All resistances are non-correlated and one TPW is used for calibration and another one for temperature measurement

Correlation matrix

$$
\boldsymbol{R}_{\boldsymbol{R}}=\boldsymbol{I}_{(3+N) \times(3+N)} .
$$

b) Resistances in DFPs are correlated and one TPW is used for calibration and another one for temperature measurement

If SPRT resistances in DFPs are correlated in calibration, which may be due to the same conditions in both DFP implementation and in measuring SPRT resistances in DFP, and one triple point is used in calibration and another in measurement, while correlations between resistances at $T$ and the TPW in temperature measurement are not assumed, the correlation matrix of input quantities $\boldsymbol{R}_{\boldsymbol{R}}$ of the $(3+N) \times$ $(3+N)$ type is

$$
\boldsymbol{R}_{\boldsymbol{R}}=\left(\begin{array}{llllll}
1 & 0 & 0 & \cdots & 0 & 0 \\
0 & 1 & 0 & \cdots & 0 & 0 \\
0 & 0 & 1 & \cdots & r\left(R_{\mathrm{DFP} 1}, R_{\mathrm{DFP} N}\right) & r\left(R_{\mathrm{DFP} 1}, R_{\mathrm{TPW} \mathrm{cal}}\right) \\
\vdots & \vdots & \vdots & \ddots & \vdots & \vdots \\
0 & 0 & r\left(R_{\mathrm{DFP} \mathrm{N}}, R_{\mathrm{DFP} 1}\right) & \cdots & 1 & r\left(R_{\mathrm{DFP} N}, R_{\mathrm{TPW} \text { cal }}\right) \\
0 & 0 & r\left(R_{\mathrm{TPW} \mathrm{cal}}, R_{\mathrm{DFP} 1}\right) & \cdots & r\left(R_{\mathrm{TPW} \mathrm{cal}}, R_{\mathrm{DFP} N}\right) & 1
\end{array}\right)
$$

\section{NUMERICAL EXAMPLE}

For the sake of illustration, a numerical example of the determination of the overall uncertainty of SPRT calibrated in the water-aluminum sub-range for the data presented in [13], [18] is given. We consider specific numerical examples of the three cases given in the previous section regarding the TPW. Assuming the use of a $25 \Omega$ SPRT, the same value of $1.17 \cdot 10^{-2} \mathrm{~m} \Omega$ for the uncertainty of the SPRT resistance in TPW is always considered. The uncertainty values of SPRT resistances in DFP are considered, being $3.85 \cdot 10^{-2} \mathrm{~m} \Omega$ for $\mathrm{Sn}$, $4.98 \cdot 10^{-2} \mathrm{~m} \Omega$ for $\mathrm{Zn}$, and $6.32 \cdot 10^{-2} \mathrm{~m} \Omega$ for $\mathrm{Al}$.

We have considered two cases of correlation between SPRT resistances for temperature measurements in and outside the calibration laboratory (for the sake of simplicity, $r=1$ is considered for correlations between resistances in TPW, i.e. we assume one TPW resistance in the calculation):

a) We do not consider the correlations between the resistances, except for the correlations between the resistances in the TPW in calibration, as mentioned above.

b) Correlations between resistances in DFP are considered: $r\left(R_{\mathrm{DFP} i}, R_{\mathrm{DFP} j}\right)=0.45, r\left(R_{\mathrm{DFP} i}, R_{\mathrm{TPW} j}\right)=0.56$ for all $i \neq$ $j$.

Fig.1. shows an example of the propagation of uncertainties in the realization of the temperature scale outside the calibration laboratory considering one resistance in TPW for different correlations between DFPs. Correlations between the resistance in temperature measurement and the resistance in DFP are not expected here. The effect of correlation between resistances in DFPs is manifested in some leveling of the uncertainty propagation curve. Individual curves intersect at DFP and at one more temperature. 


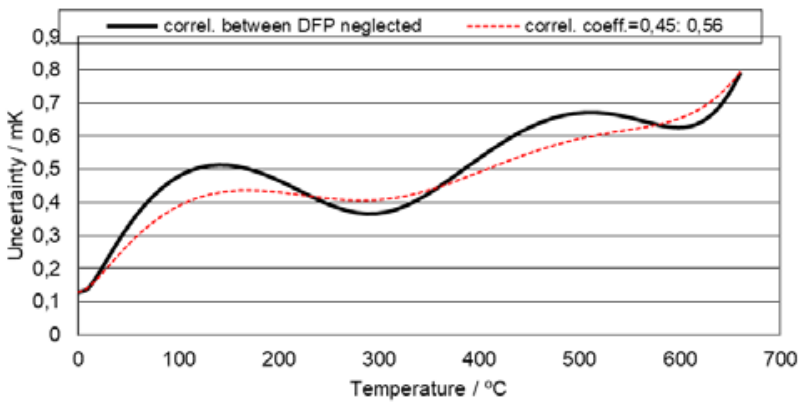

Fig.1. Temperature measurement outside a calibration laboratory with a TPW from calibration, $r\left(R_{\mathrm{TPW}}, R_{\mathrm{TPW} j}\right)=1$. Correlations between resistances in temperature measurement and during calibration are not considered. Covariances between resistances in calibration are considered, $\quad r\left(R_{\mathrm{DFP} i}, R_{\mathrm{DFP} j}\right)=0.45$, $r\left(R_{\mathrm{DFP} i}, R_{\mathrm{TPW} j}\right)=0.56$.

Fig.2. shows an example of propagation of uncertainties in the realization of the temperature scale outside the calibration laboratory, when the client uses their own resistance in TPW for different correlations between DFPs during calibration. Correlations between the temperature measurement resistance and the resistance in DFP are not expected here. The effect of the correlation between the resistances in DFP will be manifested by some reduction of the uncertainties of temperature determination.

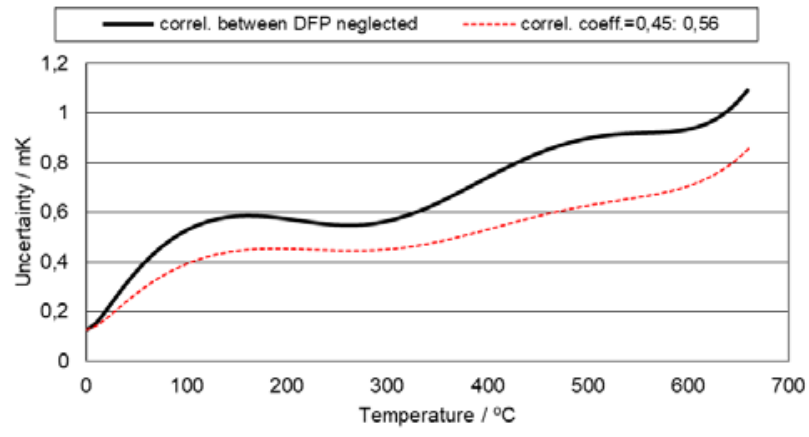

Fig.2. Temperature measurement outside a calibration laboratory, with client's own resistance in TPW, $r\left(R_{\mathrm{TPW}}, R_{\mathrm{TPW} j}\right)=0$. Correlations between resistances in temperature measurement and during calibration are not considered. Covariances between resistances in calibration are considered, $r\left(R_{\mathrm{DFP} i}, R_{\mathrm{DFP} j}\right)=0.45$, $r\left(R_{\mathrm{DFP} i}, R_{\mathrm{TPW} j}\right)=0.56$.

Fig.3. shows an example of the propagation of uncertainties in the realization of the temperature scale in a calibration laboratory with one TPW, considering the correlations between the resistances in DFP both in calibration and in temperature measurement. The correlations cause a decrease in the uncertainty of the temperature scale realization (temperature measurement).

An important conclusion is that covariance between SPRT resistances in DFPs can have a significant effect on the magnitude of temperature measurement uncertainty. Their contribution may be greater than the contribution of the covariances between the relative resistances caused by using the same SPRT resistance in the TPW for calibration, respectively for measurement and calibration. The graphs show a typical data situation where the real correlations between SPRT resistances in DFP were characterized by correlation coefficients between DFPs of around 0.3; or 0.45 between DFP and TPW. We also considered the case of their neglect $(r=0)$, respectively full correlation $(r=1)$ for all uses of SPRT in TPW for calibration and measurement.

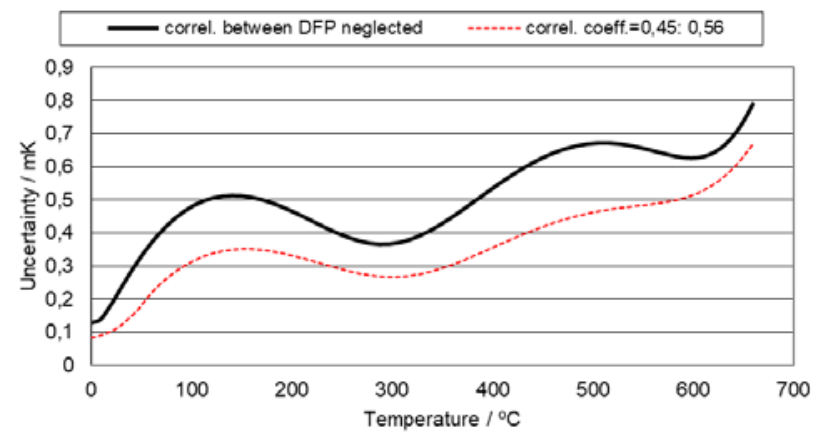

Fig.3. Temperature measurement in a calibration laboratory. One TPW, resistance measurement in temperature measurement in laboratory is correlated with resistance obtained during calibration, $r\left(R_{\mathrm{DFP} i}, R_{\mathrm{DFP} j}\right)=0.45, r\left(R_{\mathrm{DFP} i}, R_{\mathrm{TPW} j}\right)=0.56$,

$r\left(R_{\mathrm{TPW}}, R_{\mathrm{TPW} j}\right)=1, \quad r\left(R, R_{\mathrm{DFPi}}\right)=0.45, r\left(R, R_{\mathrm{TPW} i}\right)=$ $r\left(R, R_{\mathrm{TPW}}\right)=0.56$.

\section{CONCLUSION}

The paper introduces a procedure for the determination of temperature scale uncertainties realized by SPRT calibrated in DFP, which was formulated by matrix algebra, presented in Supplement 2 of GUM. As Rosenkranz [9] has shown, this method has many advantages, it is simple and accurate. In this paper, however, we do not proceed from relative resistances, but directly from the resistances obtained during calibration and/or measurement. This enables including any correlation between calibration and measurement resistance in a very simple way. For this procedure it is enough to determine the covariance matrix of resistances and the output provides the resulting uncertainty of temperature measurement. Sensitivity coefficient matrices do not change; only correlation or covariance matrices of resistances in DFPs for calibration and temperature measurements change. The paper shows the possibility of some simplification of input covariance (correlation) matrices if we distinguish measurements in or outside a calibration laboratory. When software for calculation with matrices is readily available, this procedure is efficient and reliable. It does not require any special knowledge.

The influence of the correlation between resistances in DFP, which the procedure shown here can simply consider in the calculation, has already been addressed in some work. In this paper we do not address the correlation balance itself (see [2] but also [10]-[13] for details), in the demonstration examples we only point out the possible effects of correlations on overall uncertainty. Also, there are no 
uncertainties due to ambiguity. It turns out that there is a difference whether a TPW resistance obtained from calibration is used in measurement or a measured TPW resistance is used in measurement. The first case is marked as a laboratory measurement and the second case as a measurement outside laboratory.

\section{ACKNOWLEDGMENT}

This research was supported by the Slovak University of Technology in Bratislava and the APVV agency (Grants No. APVV-0295-15, APVV-18-0066), the VEGA agency (Grants No. 1/0098/18, No. 1/0556/17), and the KEGA agency (Grants No. 0065STU-4/2018 and No. 039STU4/2017).

\section{REFERENCES}

[1] BIPM. (2018). Guide to the Realisation of the ITS-90. https://www.bipm.org/en/committees/cc/cct/guideits90.html.

[2] White, D.R., Ballico, M., Chimenti, V., Duris, S., Filipe, E., Ivanova, A., Kartal Dogan, A., MendezLango, E., Meyer, C., Pavese, F., Peruzzi, A., Renaot, E., Rudtsch, S., Yamazawa, K. (2009). CCT/08-19/rev. BIPM. http://www.bipm.org/cc/CCT/Allowed/24/D19 _rev_WG3_Doc_rev_10July2009.pdf.

[3] White, D.R., Saunders, P. (2000). Propagation of uncertainty on interpolated scales, with examples from thermometry. Metrologia, 37, 285-293.

[4] White, D.R. (2001). The propagation of uncertainty with non-Lagrangian interpolation. Metrologia, 38 (1), 63.

[5] White, D.R., Saunders, P. (2007). The propagation of uncertainty with calibration equations. Measurement Science Technology, 18 (7), 2157.

[6] White, D.R., Strouse, G.F. (2009). Observations on subrange inconsistency in the SPRT interpolations of ITS90. Metrologia, 46 (1), 101.

[7] Joint Committee for Guides in Metrology. (2008). Evaluation of measurement data - Guide to the expression of uncertainty in measurement. JCGM 100:2008. https://www.bipm.org/utils/common/ documents/jcgm/JCGM_100_2008_E.pdf.

[8] Joint Committee for Guides in Metrology. (2011). Evaluation of measurement data - Supplement 2 to the "Guide to the expression of uncertainty in measurement" - Extension to any number of output quantities. JCGM 102:2011. http://www.bipm.org/ utils/common/documents/jcgm/JCGM_102_2011_E.p df.

[9] Rosenkranz, P. (2011). Uncertainty propagation for platinum resistance thermometers calibrated according to ITS-90. International Journal of Thermophysics, 32 (1-2), 106-119.
[10] Duris, S., Palencar, R. (2005). The influence of covariances on the uncertainty of temperature measurement by resistance thermometer. Measurement Science Review, 5 (2), 15-18.

[11] Duris, S., Palencar, R., Ranostaj, J. (2008). Contribution of the SPRT calibration to uncertainty of temperature T90 measured by the calibrated SPRT. Measurement Science Review, 8 (1), 5-10.

[12] Duris, S., Palencar, R., Ranostaj, J. (2008). The effect of covariance on uncertainty when constructing the ITS-90 temperature scale. Measurement Techniques, 51 (4), 412-420.

[13] Duris, S., Palencar, R., Ranostaj, J. (2011). Conclusion and some comments on the calculation of uncertainty when constructing a temperature scale. Measurement Techniques, 54 (8), 910-920.

[14] Bloembergen, P. (1995). On the propagation of the uncertainty at the triple point of water, associated with the calibration of SPRTs according to the ITS-90: A case study. Metrologia, 32 (4), 253-257.

[15] Sadli, M., Renaot, E., Bonnier, G. (1998). In Proceedings of Euromet Workshop in Temperature. Paris: BNM-INM, 13-18.

[16] Meyer, C.W., Ripple, D.C. (2006). Determination of the uncertainties for ITS-90 realization by SPRTs between fixed points. Metrologia, 43 (5), 327-340.

[17] Lira, I., Camarano, D., Paredes Villalobos, J., Santiago, F. (1999). Expression of the uncertainty of measurement in the calibration of thermometers. Part I: Standard platinum resistance thermometers. Metrologia, 36, 107.

[18] Palencar, R., Duris, S., Brdecka, R. (2000). CCT/200023. CCT Working Document. BIPM.

[19] Dyurish, S., Palenchar, R. (2006). A matrix interpretation of the estimate of the extension of uncertainties when constructing a temperature scale. Measurement Techniques, 49 (7), 689-696.

[20] Palencar, R., Duris, S., Durisova, Z., Brokes, V., Pavlasek, P. (2016). Reduction of measurement uncertainty by taking into account correlation in measurements and temperature scale realization. Measurement Techniques, 59 (1), 52-58.

[21] Palencar, R., Duris, S. (2012). Matrix interpretation of the uncertainty propagation for the ITS-90 realisation. In 20th IMEKO World Congress 2012. ISBN 978-142748-190-8.

[22] Preston-Thomas, H. (1990). The International Temperature Scale of 1990 (ITS-90). Metrologia, 27, 3-10 (Erratum p. 107).

Received November 11, 2019 Accepted April 24, 2020 